\title{
Intraoperative Blood Transfusion Contributes to Decreased Long-Term Survival of Patients with Esophageal Cancer: Comments on Regression Model Estimation: Reply
}

\author{
Yoshihiro Komatsu • Hajime Orita • \\ Mutsumi Sakurada • Hiroshi Maekawa • \\ Toshitaka Hoppo • Koichi Sato
}

Published online: 20 June 2012

(c) Société Internationale de Chirurgie 2012

To the Editor

We are grateful to Fancesco and colleagues for their thoughtful comments on our recent article, Intra-operative Blood Transfusion Contributes to Decreased Long-term Survival of Patients with Esophageal Cancer [1]. We entirely agree with their comments that it was inappropriate to perform a multivariate Cox proportional hazard regression analysis by introducing ten explanatory variables when the sample size was small $(n=37)$, and to include both intraoperative blood loss and blood transfusion as explanatory variables in the multivariate analysis due to their high correlation.

We performed the Kendall Tau correlation test, which demonstrated a high correlation between intraoperative blood loss and intraoperative blood transfusion (correlation coefficient 0.535). Excluding intraoperative blood transfusion, we introduced four possible explanatory variables, including intraoperative blood loss, stage of the disease, age, and sex in the multivariate Cox proportional hazard regression analysis (Table 1). The stage of the disease and intraoperative blood loss were shown to be two independent risk factors for reduced survival $(p=0.001$ and 0.002 , respectively), whereas the other variables did not reach statistical significance. For these reasons, reanalysis using

Y. Komatsu $(\bowtie) \cdot$ H. Orita · M. Sakurada · H. Maekawa ·

K. Sato

Department of Surgery, Juntendo Shizuoka Hospital, Juntendo

University School of Medicine, 1129 Nagaoka, Izunokuni-shi,

Shizuoka 410-2295, Japan

e-mail: hirok94@gmail.com

T. Hoppo

Department of Cardiothoracic Surgery, University of Pittsburgh

Medical Center, Suite 715, 5200 Center Avenue, Pittsburgh,

PA 15232, USA
Table 1 Multivariate Cox proportional hazard regression analysis

\begin{tabular}{llll}
\hline Variables & $\begin{array}{l}\text { Hazard } \\
\text { ratio }\end{array}$ & $\begin{array}{l}95 \% \text { Confidence } \\
\text { interval }\end{array}$ & $p$ Value \\
\hline $\begin{array}{l}\text { Stage of the disease } \\
\text { Intraoperative blood } \\
\quad \text { loss }\end{array}$ & 1.637 & $0.663-1.049$ & $0.001^{*}$ \\
Age & 1.095 & $0.993-1.207$ & $0.002^{*}$ \\
Sex & 0.507 & $0.096-2.683$ & 0.069 \\
\hline
\end{tabular}

$* p<0.05$ is considered significant

the reduced number of variables did not change our conclusions and consistently demonstrated that intraoperative blood loss is an independent prognostic factor in this setting.

However, the present study is a non-randomized retrospective study involving a relatively small number of patients $(n=37)$ with a short-term follow-up (mean 3.3 years). To evaluate the actual role of intraoperative blood loss in patients with esophageal cancer, a prospective, randomized controlled study with a larger number of subjects and longer-term follow-up will be required.

Conflict of interest None.

\section{References}

1. Komatsu Y, Orita H, Sakurada M et al (2012) Intraoperative blood transfusion contributes to decreased long-term survival of patients with esophageal cancer. World J Surg 36:844-850. doi:10.1007/ s00268-012-1433-3 